TITLE:

\title{
Strange scaling and relaxation of finite-size fluctuation in thermal equilibrium
}

$\operatorname{AUTHOR}(S)$ :

Yamaguchi, Yoshiyuki Y.

\section{CITATION:}

Yamaguchi, Yoshiyuki Y.. Strange scaling and relaxation of finite-size fluctuation in thermal equilibrium. Physical Review E 2016, 94(1):

012133.

ISSUE DATE:

2016-07-22

URL:

http://hdl.handle.net/2433/216149

RIGHT:

(C)2016 American Physical Society 
PHYSICAL REVIEW E 94, 012133 (2016)

\title{
Strange scaling and relaxation of finite-size fluctuation in thermal equilibrium
}

\author{
Yoshiyuki Y. Yamaguchi* \\ Department of Applied Mathematics and Physics, Graduate School of Informatics, Kyoto University, 606-8501 Kyoto, Japan
}

(Received 10 March 2016; published 22 July 2016)

\begin{abstract}
We numerically exhibit two strange phenomena of finite-size fluctuation in thermal equilibrium of a paradigmatic long-range interacting system having a second-order phase transition. One is a nonclassical finite-size scaling at the critical point, which differs from the prediction by statistical mechanics. With the aid of this strange scaling, the scaling theory for infinite-range models conjectures the nonclassical values of critical exponents for the correlation length. The other is relaxation of the fluctuation strength from one level to another in spite of being in thermal equilibrium. A scenario is proposed to explain these phenomena from the viewpoint of the Casimir invariants and their nonexactness in finite-size systems, where the Casimir invariants are conserved in the Vlasov dynamics describing the long-range interacting systems in the limit of large population. This scenario suggests appearance of the reported phenomena in a wide class of isolated long-range interacting systems.
\end{abstract}

DOI: 10.1103/PhysRevE.94.012133

\section{INTRODUCTION}

Studying fluctuation is one of the central issues in large systems. Physical importance of fluctuation can be found in Johnson-Nyquist noise [1,2], fluctuation-induced tunneling $[3,4]$, and the fluctuation theorem [5-7]. The fluctuation is also investigated in mathematical models of $X Y$ rotors on networks [8] and of coupled oscillators [9]. In this article we concentrate on finite-size fluctuation in isolated Hamiltonian systems having long-range interaction, which we call long-range systems, and include self-gravitating systems, plasmas, two-dimensional fluids, and so on (see Refs. [10-12], for instance). We numerically reveal a strange scaling and relaxation of finite-size fluctuation in thermal equilibrium of a long-range system. We stress that the latter relaxation means that the strength of fluctuation is not constant but temporally grows from one level to another in spite of being in thermal equilibrium. In order to describe a possible mechanism, we start from sketching the relaxation process in long-range systems.

A remarkable dynamics in long-range systems is the appearance of the so-called quasistationary state (QSS), whose lifetime diverges as the population of the system increases [13-15]. The QSSs are widely observed in nature: Galaxies [16] and the great red spot of Jupiter [17] are considered examples of QSSs. Temporal evolution of a long-range system is governed by the Vlasov equation, or the collisionless Boltzmann equation, in the limit of large population [18-20], and QSSs are interpreted as stable stationary solutions to the Vlasov equation $[14,15]$ which are possibly out of equilibrium. The appearance of QSSs is explained by the existence of the Casimir invariants, which are infinite numbers of invariants of the Vlasov dynamics. We note that the Casimir invariants are written in the form $\int c(f(q, p)) d q d p$, with any differentiable function $c$ and the one-particle distribution function $f(q, p)$ on the one-particle phase space of position $q$ and momentum $p$. Finite-size effects play the role of collision, and the collision term drives the system from a QSS to thermal equilibrium

*yyama@amp.i.kyoto-u.ac.jp by breaking the Casimir constraints. We remark that $N$-body systems are exactly described by a Vlasov-type equation, the Klimontovich equation [10], but the Casimir-type functionals lose the role of invariants. Indeed, in the Klimontovich equation, the distribution function $f(q, p)$ consists of the sum of Dirac's $\delta$ functions, and, for instance, $\int[f(q, p)]^{2} d q d p$ diverges due to squares of the $\delta$ functions.

QSSs are observed even applying an external force to the system. The external force drives the system from an initial state, for instance a thermal equilibrium state, to a QSS before going towards a forced thermal equilibrium state. For the system having a second-order phase transition, the long lifetime of QSSs makes it possible to define the critical exponents for the response in QSSs, $\gamma_{ \pm}$and $\delta$, around and at the critical point, respectively. Hereafter, the subscript $+(-)$ indicates that the variable is defined in the disordered (ordered) phase. Linear [21,22] and nonlinear [23-25] response theories based on the Vlasov description reveal that $\gamma_{+}=1$ but $\gamma_{-}=$ $1 / 4$ [26] and $\delta=3 / 2[23,24]$ in the Hamiltonian mean-field (HMF) model [27,28], while equilibrium statistical mechanics gives $\gamma_{ \pm}=1$ and $\delta=3$. The former nonclassical critical exponents are again explained by the Casimir constraints, which suppress the response.

We note that the strange critical exponents for the response are obtained not in the forced thermal equilibrium but in QSSs. However, if the fluctuation-response relation holds, then anomalous fluctuation appears in nonforced thermal equilibrium. Similarly, relaxation of the strength of finite-size fluctuation can be expected since the nonclassical values of $\gamma_{-}$ and $\delta$ come from the Casimir constraints, but the finite-size effects slowly release the system from the constraints as the sketched relaxation process.

To examine the above scenario, we perform direct $N$-body simulations in the HMF model. We remark that validity of the fluctuation-response relation is not obvious since the response theories [21-25] are based on the Vlasov dynamics in which the limit of large population is taken. We therefore first confirm the fluctuation-response relation for off-critical points, and report the strange scaling at the critical point corresponding to the nonclassical critical exponent $\delta=3 / 2$. After that, we show that the strength of finite-size fluctuation relaxes from 
one level to another in thermal equilibrium. We underline that, since the scenario is based on the existence of the Casimir invariants, the reported phenomena are not limited in the HMF model, but can be expected in generic long-range systems. In addition, we discuss that the scaling theory [29-31] conjectures nonclassical values of critical exponents for the correlation length with the aid of the strange scaling.

This article is constructed as follows. We introduce the HMF model in Sec. II with a short review of results by statistical mechanics. In Sec. III the two main phenomena are reported by performing direct $N$-body simulations in the HMF model, and a scenario based on the Casimir invariants is proposed to understand the phenomena. We consider the scaling theory in Sec. IV, which conjectures the critical exponents for correlation length. The final section (Sec. V) is devoted to a summary and discussions.

\section{THE MODEL}

The HMF model is a paradigmatic long-range system and is expressed by the $N$-body Hamiltonian

$$
H_{N}=\sum_{j=1}^{N} \frac{p_{j}^{2}}{2}+\frac{1}{2 N} \sum_{j, k=1}^{N}\left(1-\boldsymbol{m}_{j} \cdot \boldsymbol{m}_{k}\right)-\boldsymbol{h} \cdot \sum_{j=1}^{N} \boldsymbol{m}_{j},
$$

where $\boldsymbol{m}_{j}=\left(\cos q_{j}, \sin q_{j}\right)$ is a $X Y$ spin and $\boldsymbol{h}=\left(h_{x}, h_{y}\right)$ is the external force. The particles are confined on the unit circle, and $q_{j} \in(-\pi, \pi]$ and $p_{j} \in \mathbb{R}$ are the phase of $j$ th particle and the conjugate momentum, respectively. The factor $1 / N$ in the potential term is added to ensure extensivity of energy. The magnetization (order parameter) vector of the HMF model is defined by the arithmetic mean of $\boldsymbol{m}_{j}$ as

$$
\boldsymbol{M}=\left(M_{x}, M_{y}\right)=\frac{1}{N} \sum_{j=1}^{N} \boldsymbol{m}_{j} .
$$

The HMF model has the second-order phase transition between the disordered phase $\left(T>T_{\mathrm{c}}\right)$ and the ordered phase $\left(T<T_{\mathrm{c}}\right)$ with the critical temperature $T_{\mathrm{c}}=1 / 2$ [28].

We denote the canonical average of $\boldsymbol{M}$ by $\langle\boldsymbol{M}\rangle$ and define the isothermal zero-field susceptibility tensor $\chi^{\mathrm{T}}=\left(\chi_{a b}^{\mathrm{T}}\right)$ as

$$
\chi_{a b}^{\mathrm{T}}=\left.\frac{\partial\left\langle M_{a}\right\rangle}{\partial h_{b}}\right|_{\boldsymbol{h}=\mathbf{0}}, \quad(a, b \in\{x, y\}) .
$$

It is straightforward to prove the relation

$$
\operatorname{tr} \chi^{\mathrm{T}}=\frac{N}{T}\left(\left\langle\boldsymbol{M}^{2}\right\rangle-\langle\boldsymbol{M}\rangle^{2}\right)
$$

for $\boldsymbol{h}=\mathbf{0}$ from the explicit expression of $\langle\boldsymbol{M}\rangle$ and the definition of $\chi^{\mathrm{T}}$. We note $\langle\boldsymbol{M}\rangle=\mathbf{0}$ from symmetry of the system. Further derivations give

$$
\left\langle\boldsymbol{M}^{2}\right\rangle=O\left(N^{-1 / 2}\right) \quad \text { at } T=T_{\mathrm{c}} .
$$

See Refs. [31,32], for instance.

The magnetization vector fluctuates around $\boldsymbol{M}=\mathbf{0}$ in the disordered phase, and there is no special direction on the twodimensional $\boldsymbol{M}$ plane. On the other hand, in the ordered phase, the Goldstone mode appears, and the zero-field susceptibility diverges for this direction [22,33]. This fact suggests to observe the amplitude of magnetization only. Taking the above discussions into account, we compute the finite-size fluctuation separately in the ordered and the disordered phases by the quantity

$$
V_{M}(N)= \begin{cases}\left\langle\boldsymbol{M}^{2}\right\rangle & \left(T \geqslant T_{\mathrm{c}}\right), \\ \left\langle\|\boldsymbol{M}\|^{2}\right\rangle-\langle\|\boldsymbol{M}\|\rangle^{2} & \left(T<T_{\mathrm{c}}\right) .\end{cases}
$$

The fluctuation-response relation is written in the form

$$
\frac{N V_{M}(N)}{T}=\epsilon \chi_{x x}^{\mathrm{T}}, \quad \epsilon= \begin{cases}2 & \left(T \geqslant T_{\mathrm{c}}\right), \\ 1 & \left(T<T_{\mathrm{c}}\right),\end{cases}
$$

where the factor $\epsilon$ comes from the dimensionality of the observables, $\boldsymbol{M}$ and $\|\boldsymbol{M}\|$, and the rotational symmetry for $T \geqslant T_{\text {c. }}$.

In the next section we perform numerical simulations without thermal noise (microcanonical setting), and hence we also introduce the isoentropic zero-field susceptibility tensor $\chi^{\mathrm{E}}$ for comparison. It is derived by considering the adiabatic process and the conservation of energy (see the Appendix for the derivation). In general, the existence of invariants suppresses the susceptibility and the isoentropic susceptibility satisfies the inequality $\chi_{x x}^{\mathrm{E}} \leqslant \chi_{x x}^{\mathrm{T}}$ accordingly [34,35].

\section{NUMERICAL RESULTS}

We numerically integrate the canonical equations of motion associated with the Hamiltonian (1) by using the fourth-order symplectic integrator [36] with the fixed time step $\Delta t=0.1$. We introduced the Hamiltonian (1) with the external field $\boldsymbol{h}$, but it is added for defining the susceptibility. In all the simulations reported hereafter, the external field is set as zero, $\boldsymbol{h}=\mathbf{0}$, although we mention the external field from the viewpoint of a response theory.

The initial values of $N$ pairs of $\left(q_{j}, p_{j}\right)$ are randomly picked up from the equilibrium one-particle distribution function

$$
f_{\mathrm{eq}}(q, p)=e^{-H(q, p) / T} / \int_{-\pi}^{\pi} d q \int_{-\infty}^{\infty} d p e^{-H(q, p) / T},
$$

where the one-particle Hamiltonian is

$$
H(q, p)=p^{2} / 2-M \cos q
$$

and $M=\|\boldsymbol{M}\|$ is the spontaneous magnetization in thermal equilibrium satisfying the self-consistent equation

$$
M=\frac{I_{1}(M / T)}{I_{0}(M / T)},
$$

with $I_{n}$ the modified Bessel functions of the first kind. For $T \geqslant T_{\mathrm{c}}$, this equation has the unique solution $M=0$, but for $T<T_{\text {c }}$, there are the solutions of $M=0$ and $M>0$. We adopt the latter by thermal stability.

We remark again that temperature $T$, appearing in Eqs. (8) and (10), just parametrizes the family of $f_{\text {eq }}$. The simulations are performed following the canonical equations of motion associated with the Hamiltonian (1) under $\boldsymbol{h}=0$, and no thermal noise is applied. We also remark that the total momentum is conserved under $\boldsymbol{h}=0$ and is zero in averages. However, the zero total momentum does not prevent magnetization $\boldsymbol{M}$ from rotating, since the center of mass of $q_{j}$ is defined on $\mathbb{R}$, but the magnetization is defined on the unit circle. For $T \geqslant T_{\mathrm{c}}$, the magnetization $\boldsymbol{M}$ actually fluctuates on the two-dimensional plane as defined in Eq. (6). 
We compute $\boldsymbol{M}(t)$ numerically and replace the canonical averages in $V_{M}(N)$ [Eq. (6)], with the time averages defined by

$$
\left\langle\boldsymbol{M}^{2}\right\rangle_{t}=\frac{1}{t_{\mathrm{av}}} \int_{t_{\mathrm{ini}}}^{t_{\mathrm{av}}+t_{\mathrm{ini}}} \boldsymbol{M}^{2}(t) d t
$$

for instance. We further take the average of $V_{M}(N)$ over 100 realizations of initial states, but denote it by $V_{M}(N)$
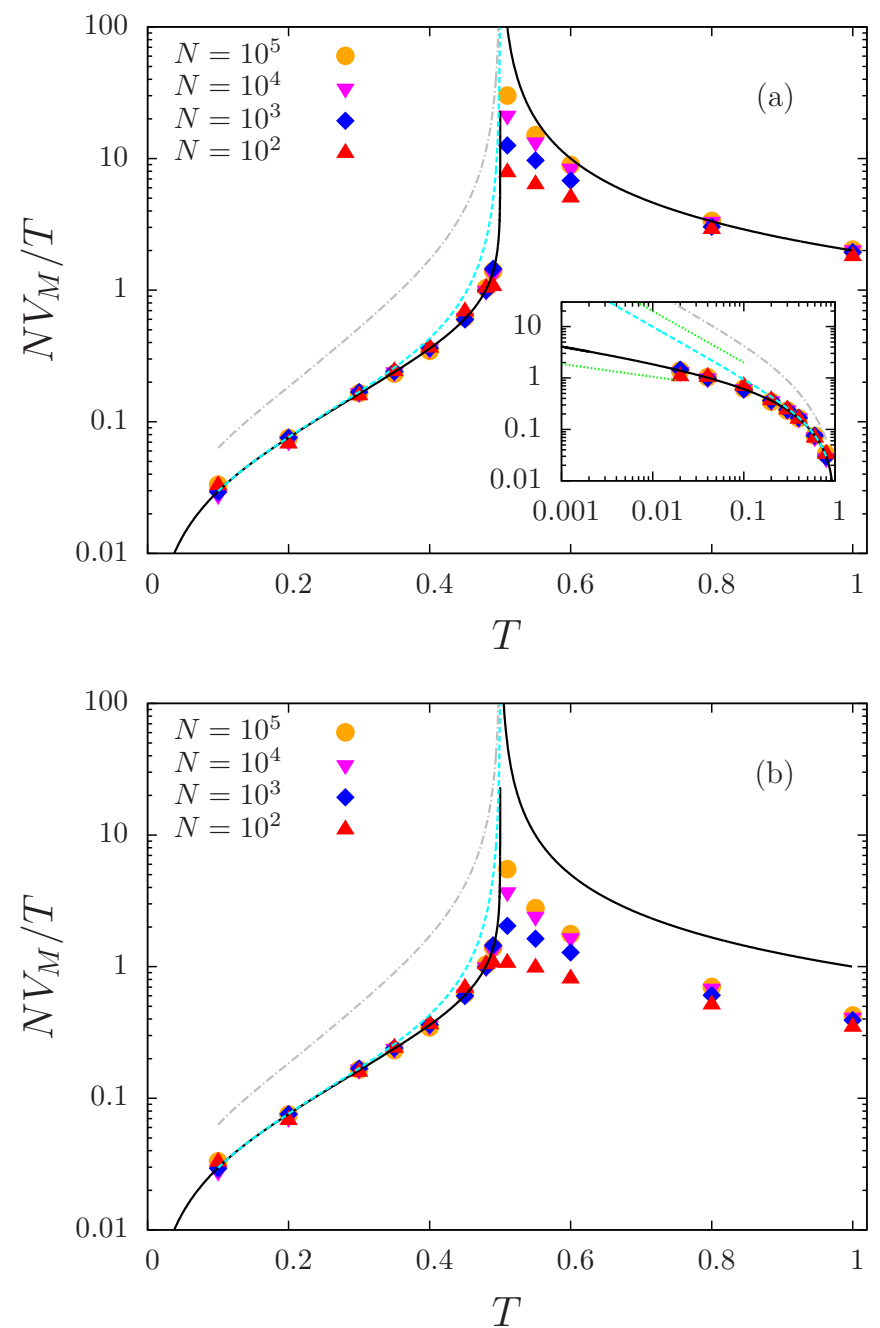

FIG. 1. Comparison between the finite-size fluctuation $N V_{M} / T$ (points) and the zero-field susceptibility $\epsilon \chi_{x x}^{\mathrm{V}}$ obtained in the Vlasov dynamics, whose level is reported by the black solid lines. The dot-dashed gray and the dashed light-blue lines in the ordered phase $\left(T<T_{\mathrm{c}}=1 / 2\right)$ are isothermal $\chi_{x x}^{\mathrm{T}}$ and isoentropic $\chi_{x x}^{\mathrm{E}}$ zero-field susceptibilities [26], respectively, while they coincide with the Vlasov one in the disordered phase. Points are averages over 100 realizations. Averaging time $t_{\mathrm{av}}$ for one realization is $t_{\mathrm{av}}=100$, and $t_{\mathrm{ini}}=0$. $N=10^{2}$ (red triangles), $10^{3}$ (blue diamonds), $10^{4}$ (magenta inverse triangles), and $10^{5}$ (orange circles). (a) $V_{M}$ is defined by Eq. (6). (Inset) Double-logarithmic graph in the ordered phase with the horizontal axis of the reduced temperature $\tau$. Slopes of the two green dotted guide lines are -1 and $-1 / 4$ from top to bottom, where the former corresponds to $\gamma_{-}=1$ for $\chi_{x x}^{\mathrm{T}}$ and $\chi_{x x}^{\mathrm{E}}$ and the latter corresponds to $\gamma_{-}=1 / 4$ for $\chi_{x x}^{\mathrm{V}}$. (b) $V_{M}$ is defined by the second line of Eq. (6) for both the ordered and the disordered phases with $\epsilon=1$. for simplicity. The initial time $t_{\text {ini }}$ will be used to examine whether the prepared initial states are in thermal equilibrium, and $t_{\text {ini }}=0$ if no special comment is given.

The finite-size fluctuation $N V_{M} / T$ is compared with the zero-field susceptibility $\chi_{x x}^{\mathrm{V}}$ obtained theoretically [22] from the Vlasov dynamics in Fig. 1(a). They are in good agreement in the ordered phase and for large $T$ in the disordered phase. Even around the critical point of the disordered phase, we can find a tendency of convergence of the fluctuation to the Vlasov susceptibility level as $N$ increases. Moreover, for the reduced temperature $\tau=\left|T-T_{\mathrm{c}}\right| / T_{\mathrm{c}}$, the scaling of $N V_{M} / T \propto \tau^{-1 / 4}$ is found in the ordered phase corresponding to the nonclassical exponent $\gamma_{-}=1 / 4$ defined by $\chi_{x x}^{\mathrm{V}} \propto \tau^{-\gamma_{-}}$, while the classical exponent is $\gamma_{-}=1$ for both $\chi_{x x}^{\mathrm{T}}$ and $\chi_{x x}^{\mathrm{E}}$ [see the inset of Fig. 1(a)]. We recall that existence of invariants suppresses the susceptibility, and the relation $\chi_{x x}^{\mathrm{T}} \geqslant \chi_{x x}^{\mathrm{E}} \geqslant \chi_{x x}^{\mathrm{V}}$ is reasonable. Thus, we conclude that the fluctuation-response relation holds even under the Casimir constraints and the finite-size effects. The validity of the definition of $V_{M}$ [Eq. (6)] is confirmed by testing the second line of (6) for the disordered phase with setting $\epsilon=1$ in the fluctuation-response relation (7). If we observe the amplitude of $\boldsymbol{M}$ only, we miss the fluctuationresponse relation in the disordered phase $T>T_{\mathrm{c}}$, as shown in Fig. 1(b).

The susceptibility diverges at the critical point due to nonlinearity of the response, and the nonlinearity is indicated by the critical exponent $\delta$. Denoting the magnetization under the external field $h=\|\boldsymbol{h}\|$ by $M_{h}$, the critical exponent is defined by $M_{h} \propto h^{1 / \delta}$. The classical value is $\delta=3$, but in the Vlasov dynamics, $\delta=3 / 2$ is obtained by a nonlinear response theory [23]. The theory derives the self-consistent equation for $M_{h}$, which induces the Landau-like pseudofree energy per one

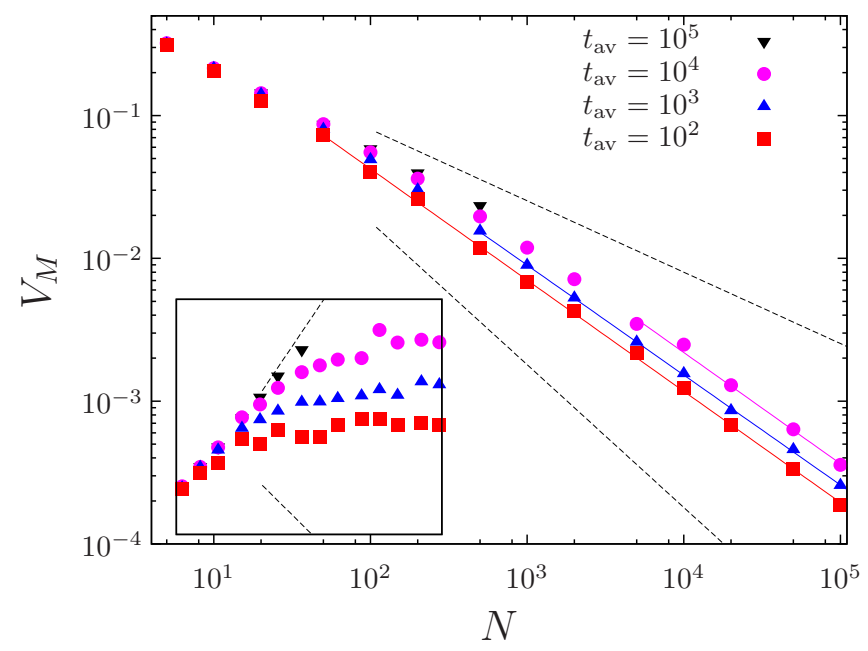

FIG. 2. Finite-size fluctuation $V_{M}$ at the critical point $T=T_{\mathrm{c}}$. Points are averages over 100 realizations. Averaging times $t_{\mathrm{av}}$ for one realization are $10^{2}$ (red squares), $10^{3}$ (blue triangles), $10^{4}$ (magenta circles), and $10^{5}$ (black inverse triangles). $t_{\text {ini }}=0$. The solid lines are computed by the least-mean square method in the displayed intervals, and their slopes are $-0.779,-0.770$, and -0.779 from bottom to top. The upper and the lower black dashed lines have the slopes $-1 / 2$ and -1 , respectively, for comparison. (Inset) The vertical axis is $V_{M} N^{4 / 5}$ to enhance difference among $t_{\mathrm{av}}$. 

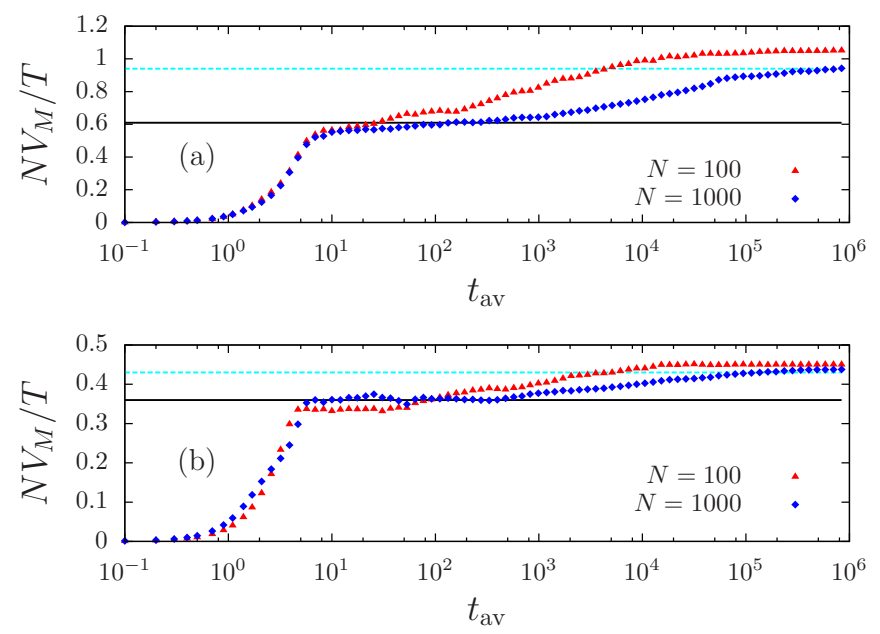

FIG. 3. Fluctuation $N V_{M} / T$ as a function of $t_{\mathrm{av}} \cdot t_{\text {ini }}=0$. (a) $T=0.45$; (b) $T=0.40$. In both panels $N=100$ (red triangles) and 1000 (blue diamonds). The black and the light-blue horizontal lines represent levels of the Vlasov and the isoentropic susceptibilities.

particle,

$$
g\left(M_{h}\right)=a\left(T-T_{\mathrm{c}}\right)\left(M_{h}+h\right)^{2}+b\left(M_{h}+h\right)^{\delta+1}-h M_{h},
$$

where $a$ and $b$ are assumed to be positive constants. In this framework a realized $M_{h}$ satisfies the equation $d g / d M_{h}=0$. We remark that the expression (12) is obtained by expanding the self-consistent equation with respect to the small external force $h$. The expansion is performed in the homogeneous disordered phase [23], and the validity of (12) is guaranteed in this phase accordingly. To make a relation between the critical exponent $\delta$ and the finite-size fluctuation at the critical point, we assume that the fluctuation level is determined by the equation $N g(M)=\Delta$ with a certain value $\Delta$. This assumption implies the scaling of $V_{M}(N) \propto N^{-2 /(\delta+1)}$ at the critical point. The classical value $\delta=3$ gives the classical scaling of (5), but the nonclassical value $\delta=3 / 2$ in the Vlasov dynamics gives the scaling of $V_{M}(N) \propto N^{-4 / 5}$. This prediction is confirmed in Fig. 2 and holds even for a rather small system as $N \simeq 100$ with a short time averaging time $t_{\mathrm{av}}=100$. We remark that the scaling tends to approach to the classical one, $N^{-1 / 2}$, as $t_{\mathrm{av}}$ increases.

Up to here we have shown that the finite-size fluctuation relates to the response. Moreover, as found in Fig. 1, there is a gap between the Vlasov and the isoentropic susceptibilities in the ordered phase. Thus, one may expect that the fluctuation, $N V_{M}(N) / T$, temporally evolves from the former level to the latter. This expectation is confirmed in Fig. 3 for $T=0.45$ and 0.40 by varying the averaging time $t_{\mathrm{av}}$. The initial increase for $t_{\mathrm{av}} \lesssim 10$ is a natural consequence from the definition of $V_{M}$ as the time average, and hence we omit it. The fluctuation is, for $N=1000$, once trapped at the Vlasov level around $t_{\mathrm{av}}=10-1000$ and goes towards the isoentropic level.

Exactly saying, the distribution function (8) represents thermal equilibrium in the limit $N \rightarrow \infty$, but the simulations
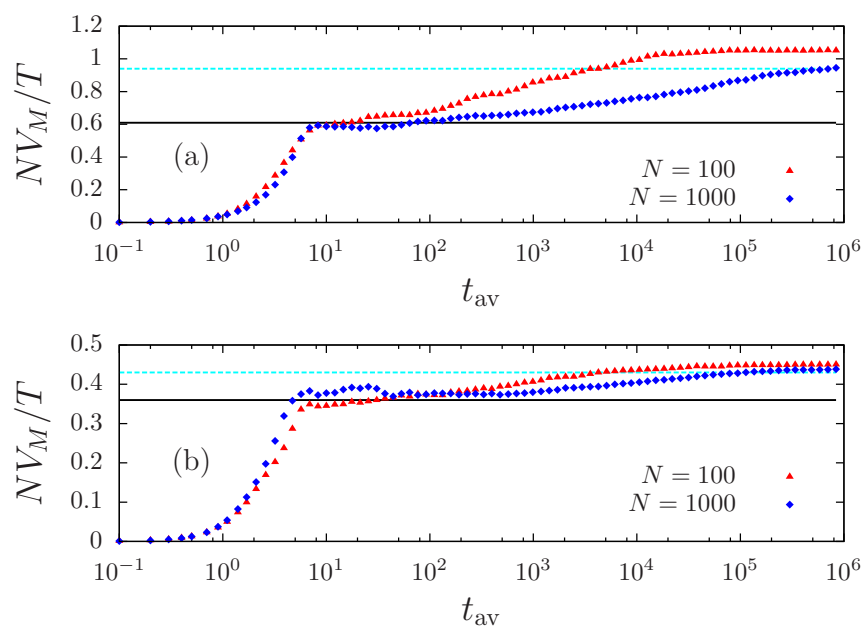

FIG. 4. The same with Fig. 3, but $t_{\text {ini }}=10^{5}$.

were performed for finite $N$. If the prepared initial states are not thermal equilibrium states but nearby nonequilibrium QSSs, then the plateaus in Fig. 3 may just correspond to the trapping in QSSs as the relaxation process sketched in Sec. I. To exclude this possibility, we change the initial time, $t_{\text {ini }}$ in (11), from 0 to $10^{5}$, where $10^{5}$ is long enough to reach thermal equilibrium even if the prepared states are the nearby QSSs (see Fig. 3). Temporal evolution with $t_{\text {ini }}=10^{5}$ is reported in Fig. 4 and is qualitatively the same as in Fig. $3\left(t_{\text {ini }}=0\right)$. We therefore conclude that the observed temporal evolution of fluctuation is not the relaxation process from nonequilibrium QSS to thermal equilibrium, but is realized in thermal equilibrium.

A qualitative change of dynamics between a short- and a long-time intervals is also captured in Fig. 5 by computing power spectra of $\boldsymbol{M}^{2}(t)$ with $N=1000$. The power spectra, each of which is the average over 100 realizations, are divided

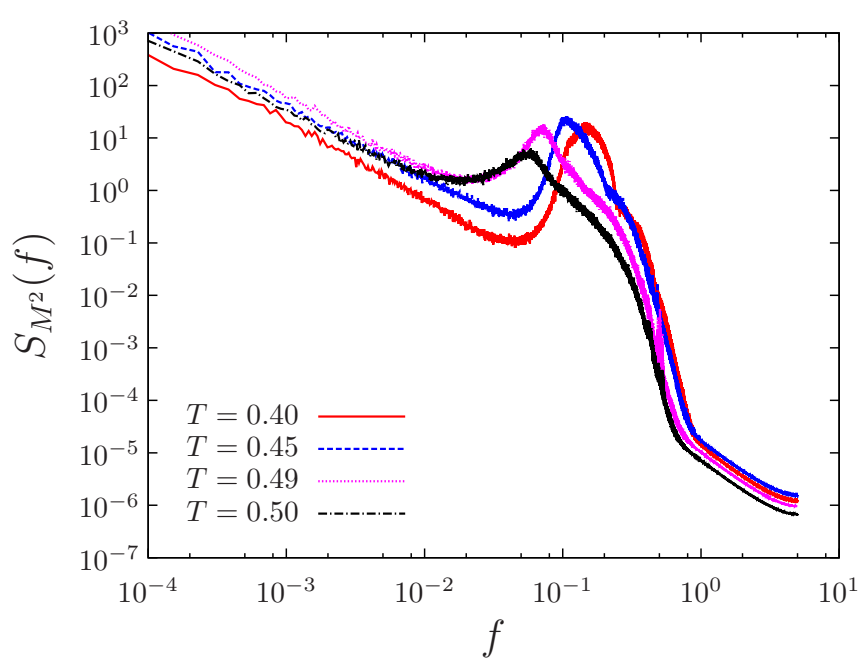

FIG. 5. Power spectra of $\boldsymbol{M}^{2}(t)$ for $T=0.40$ (red solid line), 0.45 (blue dashed line), 0.49 (magenta dotted line), and 0.50 (black dot-dashed line) with $N=1000$. Power spectra for $T>T_{\mathrm{c}}$ are also computed up to $T=0.60$ (not shown), and they are similar with for $T=0.50$ except for slight changes of slopes in a small $f$ region. 
into the short-time interval corresponding to $f \gtrsim 0.01$ and the long-time one having algebraic dampings as $f^{-a}$ with $a$ from 1.0 to 1.4 .

Putting all together, we can now make a scenario of the finite-size fluctuation in the long-range systems. In the limit $N \rightarrow \infty$, the infinite Casimir invariants divide the phase space into their level sets, and any initial state belongs to one of them and cannot escape from the level set. When $N$ is finite, invariance of the Casimir invariants is no longer exact but approximate. Nevertheless, in the short-time interval, the system fluctuates along the pseudolevel set, which induces the suppressed fluctuation, as $V_{M} \sim N^{-4 / 5}$ at the critical point. As time goes on, the system is released from the pseudoconstraints, and the classical scaling $V_{M} \sim N^{-1 / 2}$ recovers in the long-time interval. Temporal evolution of the finite-size fluctuation can be understood as the releasing process from the iso-Casimir contour. In other words, even in thermal equilibrium, the long-range systems have a kind of relaxation process from constrained states (corresponding to QSSs in thermal equilibrium) to unconstrained states. An important remark is that this process is not special: Any initial equilibrium states must evolve under the pseudo- but unavoidable Casimir constraints.

\section{SCALING THEORY}

We further investigate the finite-size fluctuation from the viewpoint of the scaling theory [29]. One hypothesis of the scaling theory is that the finite-size scalings are controlled by the dimensionless quantity $\xi / L$, where $\xi$ and $L$ are the correlation length and the system length, respectively. The infinite-range models, including the HMF model, have no concept of both lengths, but a scaling theory has been proposed for such models by replacing $\xi / L$ with $N_{\mathrm{c}} / N$ [30,31], where $N_{\mathrm{c}}$ is the coherent number. It is supposed that $N_{\mathrm{c}} \propto \xi^{d_{u}} \propto \tau^{-d_{u} v}$ with $d_{u}$ the upper critical dimensionality and $v$ defined by $\xi \propto$ $\tau^{-v}$ for the mean-field universality class. In other words, $N_{\mathrm{c}}$ corresponds to the number of particles within the correlation length $\xi$. In this setting, $v^{*}=d_{u} v$ plays the role of $v$, and the scaling function for $\chi_{x x}$, denoted by $F_{\chi}$, can be introduced as

$$
\chi_{x x}=N^{\gamma / v^{*}} F_{\chi}\left(\tau N^{1 / v^{*}}\right) .
$$

Combining the above expression with the fluctuation-response relation (7), we have

$$
\frac{N^{1-\gamma / v^{*}} V_{M}(N)}{T}=\epsilon F_{\chi}\left(\tau N^{1 / v^{*}}\right) .
$$

The scaling (14) is examined in Fig. 6(a) for the disordered phase, and the scaling function $F_{\chi}$ can be observed. In the ordered phase, the susceptibility $\chi_{x x}$ scales as $\chi_{x x} \propto \tau^{-\gamma_{-}}$, but this scaling is not excellent even for rather small $\tau$, and observation of the scaling function is hard accordingly. The susceptibility is represented as $\chi_{x x}=(1-D) / D$ [22], which defines $D$, and $D$ shows a beautiful scaling as shown in the inset of Fig. 6(b). We therefore examine the scaling for $D$, which suggests

$$
\frac{N^{\gamma_{-} / \nu_{-}^{*}}}{N V_{M}(N) / T+1}=F_{D}\left(\tau N^{1 / \nu_{-}^{*}}\right) .
$$
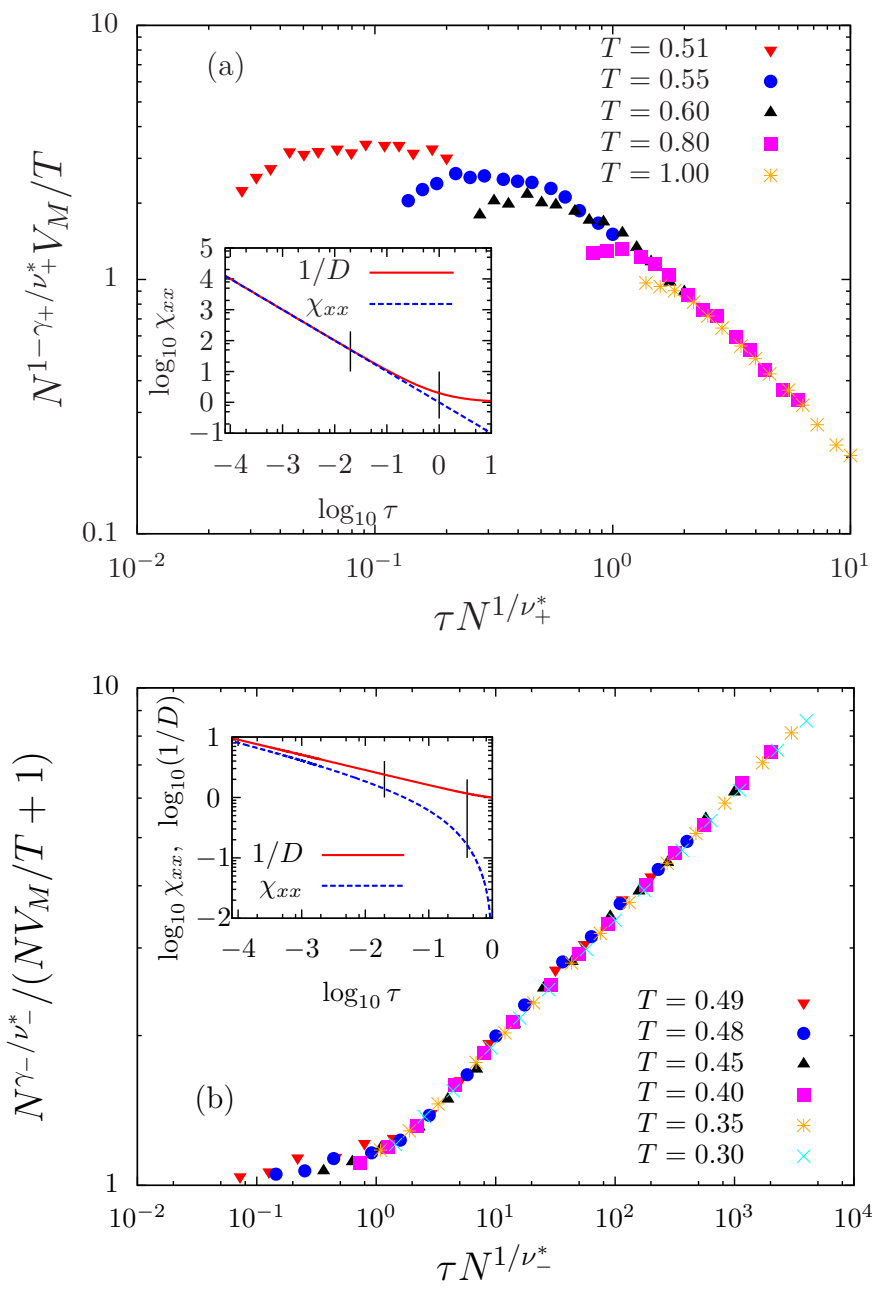

FIG. 6. Examinations of the scaling theory. (a) Disordered phase; (b) ordered phase. (Insets) $\chi_{x x}$ for the Vlasov dynamics (blue dashed lower lines) and $1 / D$ (red solid upper lines). The vertical black segments mark the computing interval of $\tau$.

The scaling function $F_{D}$ is clearly observed in Fig. 6(b), and hence we may also expect the scaling function $F_{\chi}$ in the ordered phase for small values of $\tau$.

The scaling theory suggests the values of critical exponents $v_{+}$and $v_{-}$for the correlation length. We showed that $V_{M}(N) \propto N^{-4 / 5}$ at the critical point $\tau=0$, and hence the relation (14) determines $\nu_{+}^{*}=5$ and $v_{-}^{*}=5 / 4$ from $\gamma_{+}=1$ and $\gamma_{-}=1 / 4$ [22], respectively. Thus, inputting $d_{u}=4$, although validity of the value is still not clear in the constrained equilibrium states (corresponding to QSSs), $v_{+}=5 / 4$ and $\nu_{-}=5 / 16$ can be conjectured, while $v=1 / 2$ in the classical mean-field theory. These exponents should be tested in the $\alpha$ HMF model [37], for instance, in which interaction depends on the distance between a pair of interacting particles. We remark that the exponent $\nu_{-}=5 / 16$ satisfies the scaling relation $\beta(1+\delta)=d_{u} \nu_{-}$with the exponents $\beta=1 / 2$ and $\delta=3 / 2$, while the scaling relation does not hold in the homogeneous disordered side. We also remark that, in the Vlasov dynamics, the Widom equality $\gamma=\beta(\delta-1)$ also holds in the ordered phase, but does not hold in the disordered phase. 


\section{SUMMARY AND DISCUSSIONS}

We investigated the finite-size fluctuation in thermal equilibrium of the HMF model. We have confirmed that the fluctuation-response relation holds even under the Casimir constraints and finite-size effects. This relation induces the strange scaling of $V_{M}(N) \propto N^{-4 / 5}$ at the critical point instead of $N^{-1 / 2}$ predicted by statistical mechanics. Further, we reported relaxation of the strength of finite-size fluctuation not to but in thermal equilibrium. These phenomena are explained by the existence of the Casimir invariants, which are pseudoinvariants in finite-size long-range systems. Therefore, these phenomena can be expected in generic systems. In addition, the strange scaling with the scaling theory conjectures critical exponents for the correlation length.

Clarifying the pseudoinvariants in finite-size systems has to be done for further confirmation of the scenario proposed in this article. One possible direction might be to analyze a finitesize system directly. For instance, such an analysis provides a remarkable time scale of $O\left(N^{2 / 5}\right)$, at which the ballistic approximation breaks down in the homogeneous phase [38].

We end this article by giving eight remarks. First, the linear and the nonlinear response theories are simply reproduced [24] by the so-called rearrangement formula [25]. It is worth extending the powerful formula for finite-size systems. Second, the evolution of finite-size fluctuation is not expected in the disordered phase, since there is no gap between the Vlasov susceptibility and the isoentropic one. Third, nonexactness of the Casimir invariants is a crucial point for the evolution. Exact invariants of the translational and the angular momenta break the equipartition of kinetic energy in small clusters, but this phenomenon is understood by inputting the invariants into statistical mechanics [39]. Fourth, spring-chain systems with hard springs [40] may have pseudoconstraints, lengths of bonds. The linked particles can be considered as models of molecules, for instance; thus, it might be interesting to investigate the finite-size fluctuation in such models. Fifth, the strange scaling is observed for a rather small $N$, say $N \simeq 100$, when the averaging time is short. This observation suggests that we need to consider the wreck of Casimir invariants even in small systems and might give a new perspective to understand more realistic systems. Sixth, it might be interesting to investigate the crossover time from the Vlasov fluctuation to the isoentropic one. The lifetime of a QSS with a finite magnetization is of $O(N)$ [41-43], and one may expect the same scaling since temporal evolution of the fluctuation is similar with the relaxation process from an initial state to equilibrium via QSSs and is observed in the ordered phase. Seventh, it might be also interesting to study the so-called $1 / f$ fluctuation observed in Fig. 5 from the viewpoint of existence of pseudoinvariants. Finally, we note that the exponent $v^{*}=5 / 4$ is numerically observed in the Kuramoto model with a deterministic choice of natural frequencies [9].

\section{ACKNOWLEDGMENTS}

The author thanks K. A. Takeuchi for suggesting to study the finite-size fluctuation. He also thanks S. Ogawa for valuable comments and Y. Elskens for noting a related work and helpful discussions on it. He acknowledges the supports of JSPS KAKENHI Grants No. 23560069 and No. 16K05472.

\section{APPENDIX: SUSCEPTIBILITIES}

We apply the external magnetic field of amplitude $h$ to the $x$ direction and derive the isothermal and the isoentropic susceptibilities for the direction, which are simply denoted by $\chi^{\mathrm{T}}$ and $\chi^{\mathrm{E}}$, respectively, by dropping the subscripts. Under the external field $h$, the magnetization $M_{h}$ is written with the susceptibility $\chi$ as

$$
M_{h}=M+h \chi+O\left(h^{2}\right)
$$

and the one-body Hamiltonian $H_{h}$ is

$$
H_{h}=p^{2} / 2-\left(M_{h}+h\right) \cos q .
$$

Suppose that the thermal equilibrium state is described by the one-body Boltzmann distribution (8) by replacing $H$ with $H_{h}$ and $T$ with the effective temperature $T_{h}$, which is always $T$ in the isothermal process, but $T_{h}=T+M_{h}^{2}-M^{2}+2 h\left(M_{h}-\right.$ $M$ ) for small $h$ in the isoentropic process due to the energy conservation. Omitting $O\left(h^{2}\right)$ terms, we have

$$
T_{h}=T+h \Delta T, \quad \Delta T= \begin{cases}0 & \text { (isothermal), } \\ 2 M \chi^{\mathrm{E}} & \text { (isoentropic). }\end{cases}
$$

The magnetization $M_{h}$ satisfies the self-consistent equation

$$
M_{h}=\frac{I_{1}\left(\left(M_{h}+h\right) / T_{h}\right)}{I_{0}\left(\left(M_{h}+h\right) / T_{h}\right)},
$$

and the susceptibility is computed by expanding the above equation with respect to the small $h$. Using the derivatives of the modified Bessel functions,

$$
I_{0}^{\prime}(z)=I_{1}(z), \quad I_{1}^{\prime}(z)=\frac{I_{0}(z)+I_{2}(z)}{2},
$$

the self-consistent equation (A4) is expanded up to the linear order as

$$
\chi=B(M / T)\left(\frac{1+\chi}{T}-\frac{M}{T^{2}} \Delta T\right)
$$

where

$$
B(z)=\frac{I_{0}(z)\left[I_{0}(z)+I_{2}(z)\right]-2\left[I_{1}(z)\right]^{2}}{2\left[I_{0}(z)\right]^{2}} .
$$

Substituting $\Delta T$ [Eq. (A3)] for each of the isothermal and the isoentropic processes, the susceptibilities are obtained as

$$
\chi^{\mathrm{T}}=\frac{B(M / T)}{T-B(M / T)}
$$

and

$$
\chi^{\mathrm{E}}=\frac{B(M / T)}{T-B(M / T)\left(1-2 M^{2} / T\right)},
$$

with the solution $M$ to the self-consistent equation (10). 
[1] J. B. Johnson, Thermal agitation of electricity in conductors, Phys. Rev. 32, 97 (1928).

[2] H. Nyquist, Thermal agitation of electric charge in conductors, Phys. Rev. 32, 110 (1928).

[3] P. Sheng, E. K. Sichel, and J. I. Gittleman, Fluctuation-Induced Tunneling Conduction in Carbon-Polyvinylchloride Composites, Phys. Rev. Lett. 40, 1197 (1978).

[4] P. Sheng, Fluctuation-induced tunneling conduction in disordered materials, Phys. Rev. B 21, 2180 (1980).

[5] D. J. Evans, E. G. D. Cohen, and G. P. Morris, Probability of Second Law Violations in Shearing Steady States, Phys. Rev. Lett. 71, 2401 (1993).

[6] J. Kurchan, Fluctuation theorem for stochastic dynamics, J. Phys. A 31, 3719 (1998).

[7] C. Jarzynski, Hamiltonian derivation of a detailed fluctuation theorem, J. Stat. Phys. 98, 77 (2000).

[8] S. De Nigris and X. Leoncini, Critical behavior of the $X Y$-rotor model on regular and small-world networks, Phys. Rev. E 88, 012131 (2013).

[9] H. Hong, H. Chaté, L.-H. Tang, and H. Park, Finite-size scaling, dynamic fluctuations, and hyperscaling relation in the Kuramoto model, Phys. Rev. E 92, 022122 (2015).

[10] A. Campa, T. Dauxois, and S. Ruffo, Statistical mechanics and dynamics of solvable models with long-range interactions, Phys. Rep. 480, 57 (2009).

[11] Y. Levin, R. Pakter, F. B. Rizzato, T. N. Teles, and F. P. C. Benetti, Nonequilibrium statistical mechanics of systems with long-range interactions, Phys. Rep. 535, 1 (2014).

[12] A. Campa, T. Dauxois, D. Fanelli, and S. Ruffo, Physics of LongRange Interacting Systems (Oxford University Press, Oxford, U.K., 2014).

[13] D. H. Zenette and M. A. Montemurro, Dynamics and nonequilibrium states in the Hamiltonian mean-field model: A closer look, Phys. Rev. E 67, 031105 (2003).

[14] Y. Y. Yamaguchi, J. Barré, F. Bouchet, T. Dauxois, and S. Ruffo, Stability criteria of the Vlasov equation and quasi-stationary states of the HMF model, Physica A (Amsterdam, Neth.) 337, 36 (2004).

[15] J. Barré, F. Bouchet, T. Dauxois, S. Ruffo, and Y. Y. Yamaguchi, The Vlasov equation and the Hamiltonian mean-field model, Physica A (Amsterdam, Neth.) 365, 177 (2006).

[16] J. Binney and S. Tremaine, Galactic Dynamics, 2nd ed. (Princeton University Press, Princeton, NJ, 2008).

[17] F. Bouchet and J. Sommeria, Emergence of intense jets and Jupiter's Great Red Spot as maximum-entropy structures, J. Fluid Mech. 464, 165 (2002).

[18] W. Braun and K. Hepp, The vlasov dynamics and its fluctuations in the $1 / N$ limit of interacting classical particles, Commun. Math. Phys. 56, 101 (1977).

[19] R. L. Dobrushin, Vlasov equations, Funct. Anal. Appl. 13, 115 (1979).

[20] H. Spohn, Large Scale Dynamics of Interacting Particles (Springer-Verlag, Heidelberg, 1991).

[21] A. Patelli, S. Gupta, C. Nardini, and S. Ruffo, Linear response theory for long-range interacting systems in quasistationary states, Phys. Rev. E 85, 021133 (2012).

[22] S. Ogawa and Y. Y. Yamaguchi, Linear response theory in the Vlasov equation for homogeneous and for inhomogeneous quasistationary states, Phys. Rev. E 85, 061115 (2012).
[23] S. Ogawa and Y. Y. Yamaguchi, Nonlinear response for external field and perturbation in the Vlasov system, Phys. Rev. E 89, 052114 (2014).

[24] S. Ogawa and Y. Y. Yamaguchi, Landau-like theory for universality of critical exponents in quasistationary states of isolated mean-field systems, Phys. Rev. E 91, 062108 (2015).

[25] Y. Y. Yamaguchi and S. Ogawa, Conditions for predicting quasistationary states by rearrangement formula, Phys. Rev. E 92, 042131 (2015).

[26] S. Ogawa, A. Patelli, and Y. Y. Yamaguchi, Non-mean-field critical exponent in a mean-field model: Dynamics versus statistical mechanics, Phys. Rev. E 89, 032131 (2014).

[27] S. Inagaki and T. Konishi, Dynamical stability of a simple model similar to self-gravitating systems, Publ. Astron. Soc. Jpn. 45, 733 (1993).

[28] M. Antoni and S. Ruffo, Clustering and relaxation in Hamiltonian long-range dynamics, Phys. Rev. E 52, 2361 (1995).

[29] M. E. Fisher and M. N. Barder, Scaling Theory for Finite-Size Effects in the Critical Region, Phys. Rev. Lett. 28, 1516 (1972).

[30] R. Botet, R. Jullien, and P. Pfeuty, Size Scaling for Infinitely Coordinated Systems, Phys. Rev. Lett. 49, 478 (1982).

[31] R. Botet and R. Jullien, Large-size critical behavior of infinitely coordinated systems, Phys. Rev. B 28, 3955 (1983).

[32] C. Kittel and H. Shore, Development of a phase transition for a rigorously solvable many-body system, Phys. Rev. 138, A1165 (1965).

[33] A. Campa and P. H. Chavanis, A dynamical stability criterion for inhomogeneous quasi-stationary states in long-range systems, J. Stat. Mech. (2010) P06001.

[34] P. Mazur, Non-ergodicity of phase functions in certain systems, Physica (Amsterdam) 43, 533 (1969).

[35] M. Suzuki, Ergodicity, constants of motion, and bounds for susceptibilities, Physica (Amsterdam) 51, 277 (1971).

[36] H. Yoshida, Construction of higher order symplectic integrators, Phys. Lett. A 150, 262 (1990).

[37] C. Anteneodo and C. Tsallis, Breakdown of Exponential Sensitivity to Initial Conditions: Role of the Range of Interactions, Phys. Rev. Lett. 80, 5313 (1998).

[38] B. V. Ribeiro, M. A. Amato, and Y. Elskens, Brownian regime of finite- $N$ corrections to particle motion in the XY Hamiltonian mean field model, Phys. Scr. 91, 084004 (2016).

[39] T. Niiyama, Y. Shimizu, T. R. Kobayashi, T. Okushima, and K. S. Ikeda, Inhomogeneity of Local Temperature in Small Clusters in Microcanonical Equilibrium, Phys. Rev. Lett. 99, 014102 (2007).

[40] T. Konishi and T. Yanagita, Slow relaxation to equipartition in spring-chain systems, J. Stat. Mech. (2010) P09001.

[41] P.-H. Chavanis, Kinetic equations for systems with long-range interactions: A unified description, J. Stat. Mech. (2010) P05019.

[42] M. Joyce and T. Worrakitpoonpon, Relaxation to thermal equilibrium in the self-gravitating sheet model, J. Stat. Mech. (2010) P10012.

[43] P. de Buyl, D. Mukamel, and S. Ruffo, Self-consistent inhomogeneous steady states in Hamiltonian mean-field dynamics, Phys. Rev. E 84, 061151 (2011). 